\title{
AUTOMAÇÃO DO ENSAIO DE COMBUSTÃO DE UM SIMULADOR DE PCI ${ }^{\star}$
}

\section{Resumo}

\author{
Matheus Neugebauer Motta ${ }^{1}$ \\ Claudia Caroline Teixeira Barbieri² \\ André da Silveira Machado ${ }^{3}$ \\ René Lúcio Rech ${ }^{4}$ \\ Eduardo Osório ${ }^{5}$ \\ Antônio Cezar Faria Vilela ${ }^{6}$
}

O alto-forno (AF) corresponde à principal rota de produção de ferro-gusa, matériaprima do aço. O maior desafio para as usinas siderúrgicas, nos dias de hoje, é a redução do consumo de coque, principal combustível utilizado no AF. Diante disso, o processo de injeção de carvão pulverizado ( $\mathrm{PCl}$ - Pulverized Coal Injection) é largamente empregado para substituir parte do coque utilizado por carvões de menor custo. A fim de estudar a combustibilidade de carvões e outros combustíveis sólidos com potencial para ser utilizado em PCl, o Laboratório de Siderurgia da Universidade Federal do Rio Grande do Sul (LaSid-UFRGS) desenvolveu um simulador capaz de avaliar os fenômenos envolvidos na combustão desses materiais em condições semelhantes às encontradas no AF, permitindo selecionar combustíveis, ou misturas desses, que sejam mais apropriados ao AF. Um dos principais diferenciais do equipamento é a possibilidade de coleta tanto de gases de combustão quanto de char (resíduo da combustão). Portanto, a eficiência de combustão pode ser avaliada dessas duas formas. Este trabalho tem por objetivos apresentar a automação do ensaio de combustão realizado pelo simulador de $\mathrm{PCl}$ do LaSid-UFRGS e mostrar alguns resultados de ensaios realizados..

Palavras-chave:Siderurgia; Alto-forno; PCl; Zona de combustão.

\section{Abstract}

\section{AUTOMATION OF THE COMBUSTION TEST OF A PCI TEST RIG}

Blast furnace $(B F)$ is the main route to pig iron production, raw material of steel. Nowadays the biggest challenge for steel mills is the reduction of coke, the main fuel used in BF. Faced with this, Pulverized Coal Injection (PCI) is widely used to replace part of the coke to use coal with lower price. In order to study the combustibility of the coal and other solid fuels with potential for use in PCl, the Iron and Steelmaking Laboratory of the Federal University of Rio Grande do Sul (LaSid-UFRGS) developed a $\mathrm{PCl}$ test rig able to evaluate the phenomena involved in the combustion of these fuels in similar conditions found in BF, allowing to select fuels, or blends, which is more appropriated to BF. One of the main features of the equipment is the possibility to collect either gas or char. Therefore, the combustion efficiency could be evaluated with both ways. This work aims to demonstrate the automation of the combustion test performed by the LaSid-UFRGS PCI rig and show some preliminary experimental results.

Keywords: Ironmaking; Blast furnace; PCl; Raceway.

\footnotetext{
Graduando em Engenharia Metalúrgica, bolsista de Iniciação Tecnológica, PPGE3M, UFRGS, Porto Alegre, RS, Brasil.

2 Química Industrial, MSc, doutoranda, PPGE3M, UFRGS, Porto Alegre, RS, Brasil.

Físico, MS, doutorando, PPGEM, UFMG, Belo Horizonte, MG, Brasil.

Engenheiro Químico, MSc, doutorando, PPGEQ, UFRGS, Porto Alegre, RS, Brasil.

Engenheiro Metalúrgico, Prof. Dr., professor, PPGE3M, UFRGS, Porto Alegre, RS, Brasil.

Engenheiro Metalúrgico, Prof. Dr. Ing., professor, PPGE3M, UFRGS, Porto Alegre, RS, Brasil.
} 


\section{INTRODUÇÃO}

A principal rota de produção de aço nas usinas siderúrgicas consiste no emprego de alto-forno (AF), que utiliza minério de ferro, carvão coqueificável e fundentes na produção de ferro-gusa, matéria prima do aço. O aumento da demanda de aço no cenário mundial acarretou um aumento da competição entre as indústrias. Dessa maneira, objetiva-se aumentar a produção de aço e ao mesmo tempo diminuir seus custos.

Para o aumento da produtividade e a redução dos custos as usinas siderúrgicas injetam carvão pulverizado através das ventaneiras dos AFs pelo processo de $\mathrm{PCl}$ (Pulverized Coal Injection). O PCI não é um processo recente, pois foi desenvolvido no século XIX e começou a ser utilizado por volta de 1970. Os combustíveis injetados eram gás natural e outros materiais com potencial redutor. Contudo, somente na década de noventa, no século passado, que o processo tornou-se comumente empregado [1].

O PCl proporciona diversos benefícios tanto na produção de ferro-gusa quanto na operação do AF. Porém, quando são utilizadas altas taxas de injeção, normalmente a partir de $150 \mathrm{~kg} /$ tgusa, certas contramedidas operacionais precisam ser implementadas para não prejudicar o funcionamento adequado do AF.

Dentre os principais benefícios do PCI, podem-se destacar:

1) redução de custos comparado à operação utilizando somente coque;

2) redução na emissão de gases poluentes;

3) melhora no controle operacional e aumento da produtividade do AF;

4) aumento da qualidade do metal quente e redução da quantidade de Silício (Si) no ferro-gusa[1;2].

Já que a combustão de carvões injetados produz energia e gases redutores para o processo, esta é a maneira mais eficiente para obter vantagens econômicas, produtivas e ecológicas.

Neste contexto foi desenvolvido o simulador de PCl do Laboratório de Siderurgia Universidade Federal do Rio Grande do Sul (LaSid-UFRGS). O equipamento representa desde a zona de injeção do carvão até a zona de combustão. Um dos principais diferenciais desse equipamento é a possibilidade de coleta tanto de gases de combustão quanto de char (resíduo da combustão). Uma das formas de avaliar a eficiência de combustão é através da análise dos gases da combustão quanto à relação $\mathrm{CO} / \mathrm{CO}_{2}$ por Cromatografia Gasosa. Outra forma é a determinação da conversão de carbono via Análise Termogravimétrica (TGA) da amostra antes e após a combustão. Este método nada mais é do que um balanço da quantidade de cinzas que entra e sai do reator.

\section{MATERIAIS E MÉTODOS}

O equipamento foi projetado e montado com uma série de válvulas solenoides e sensores de temperatura e pressão interligados através de tubulações de aço inoxidável refratário [3]. Como o equipamento é dividido em regiões de diferentes temperaturas e pressões a disposição das válvulas e sensores é variável ao longo do equipamento, como mostra na Figura 1.

Para melhor entendimento do mecanismo e funcionamento do equipamento são apresentadas na Tabela 1 as abreviaturas das válvulas e sensores que compõem 0 simulador do LaSid-UFRGS. 
Tabela 1 - Tabela de abreviaturas

\begin{tabular}{|l|l|}
\hline CFM20 - Controlador de vazão mássico de $\mathrm{N}_{2} ;$ & VE65 - Válvula esfera - inserção da amostra; \\
\hline CFM21 - Controlador de vazão mássico de $\mathrm{O}_{2} ;$ & $\begin{array}{l}\text { VS06 - Válvula solenoide - gás 1, painel de } \\
\text { gases; }\end{array}$ \\
\hline $\begin{array}{l}\text { CP - Combustível sólido pulverizado (carvão } \\
\text { pulverizado); }\end{array}$ & $\begin{array}{l}\text { VS07 - Válvula solenoide - gás 2, painel de } \\
\text { gases; }\end{array}$ \\
\hline FCA75 - Filtro sintetizado coletor de amostra; & $\begin{array}{l}\text { VS50 - Válvula solenoide - entrada simulador, } \\
\text { pré-aquecimento; }\end{array}$ \\
\hline $\begin{array}{l}\text { IP60 - Indicador de pressão da zona de alta } \\
\text { pressão; }\end{array}$ & $\begin{array}{l}\text { VS60 - Válvula solenoide - entrada simulador, } \\
\text { região de alta pressão; }\end{array}$ \\
\hline $\begin{array}{l}\text { IP65 - Indicador de pressão da zona de baixa } \\
\text { pressão; }\end{array}$ & $\begin{array}{l}\text { VS65 - Válvula solenoide - pulso pressão alta, } \\
\text { amostra; }\end{array}$ \\
\hline $\begin{array}{l}\text { IP75 - Indicador de pressão da zona de baixa } \\
\text { pressão; }\end{array}$ & $\begin{array}{l}\text { VS77 - Válvula solenoide - pulso, região de } \\
\text { vácuo e coleta de gases; }\end{array}$ \\
\hline $\begin{array}{l}\text { IP80 - Indicador de pressão de controle da } \\
\text { bomba de vácuo; }\end{array}$ & $\begin{array}{l}\text { VS80 - Válvula solenoide - válvula de controle de } \\
\text { vácuo. }\end{array}$ \\
\hline $\begin{array}{l}\text { IT72 - Indicador de temperatura da região de } \\
\text { mistura gás-sólido (carvão com gás quente); }\end{array}$ & \multicolumn{2}{|l}{} \\
\hline
\end{tabular}

\subsection{Design}

O simulador de PCI do LaSid-UFRGS foi projetado com o objetivo de simular a injeção de carvão em AF em escala laboratorial. Os diferenciais desse projeto são a automação com aquisição de dados em alta velocidade, o controle flexível dos parâmetros operacionais e a coleta tanto dos gases de combustão quanto de char. Sua construção vertical possibilita uma menor perda de pressão e operação mais estável [4]. O projeto do simulador baseou-se, inicialmente, em modelos de simuladores já existentes, sobretudo no simulador do IEHK-RWTH, em Aachen, na Alemanha [5].

A Figura 1 apresenta o esquema do simulador de PCl do LaSid-UFRGS. O simulador foi construído em tubulação de aço inoxidável refratário SS 310 schedule 40, para suportar pressões de até $1300 \mathrm{kPa}$ e temperaturas até $1300 \mathrm{~K}$ [3]. $\mathrm{O}$ equipamento é composto por dois fornos, um para pré-aquecimento e outro para combustão, um conjunto de válvulas eletromagnéticas, sensores de pressão e temperatura e equipamentos para supervisão, controle e aquisição de dados. 


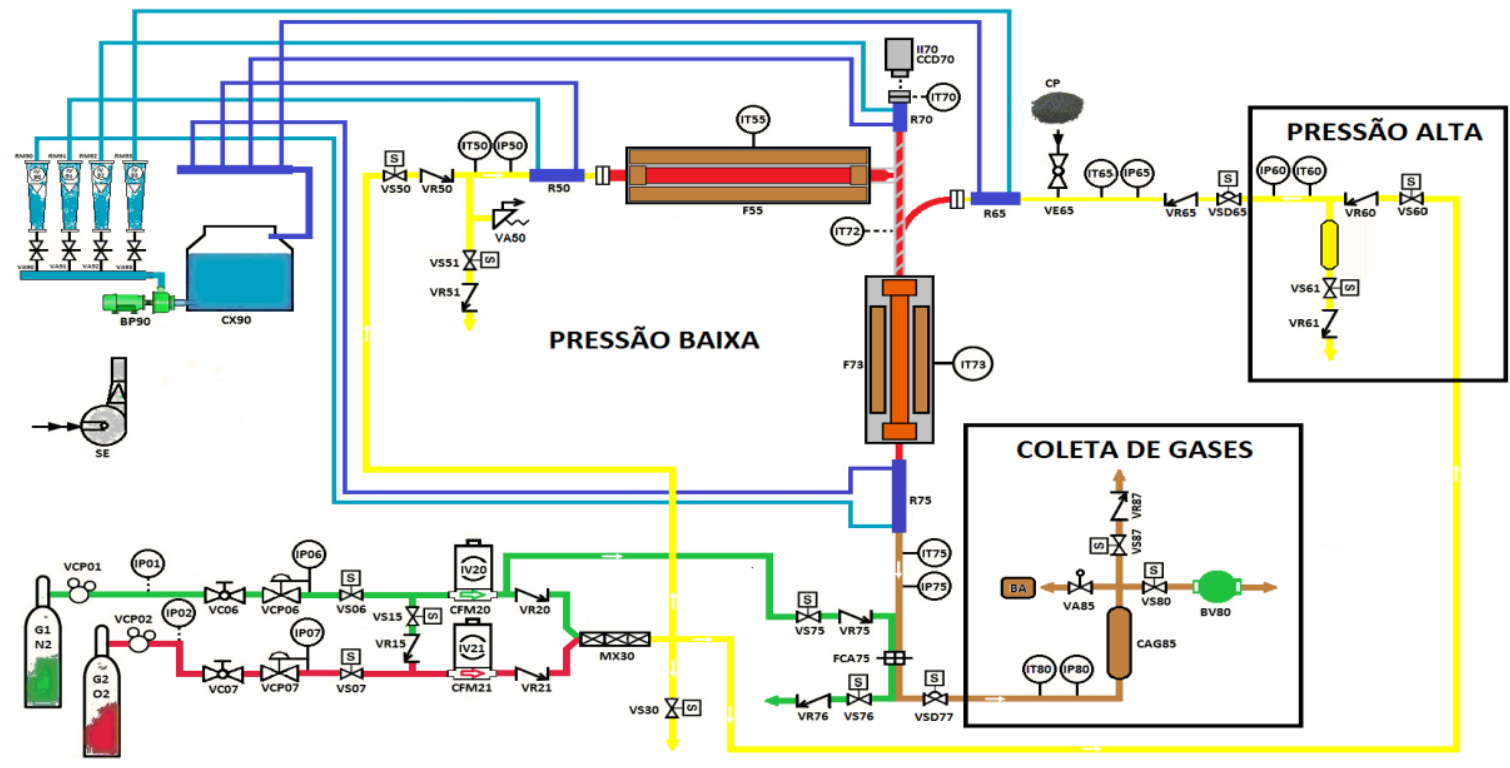

Figura 1 - Esquema do simulador de PCI do LaSid-UFRGS

Os fornos de pré-aquecimento e combustão estão posicionados respectivamente na posição horizontal e vertical, dessa maneira é permitido avaliar com mais eficiência o perfil térmico das temperaturas dentro do forno de combustão e ao longo do eixo de combustão, onde ocorrem o aquecimento, a desvolatilização e a combustão do material injetado. Isto permite a leitura da temperatura no ponto de encontro da mistura de combustível sólido com o gás pré-aquecido, continuamente [3]. Durante um ensaio a amostra é impulsionada da zona de injeção através das zonas de préaquecimento e de combustão pelo gás pressurizado da região de pressão alta. $A$ amostra sofre aquecimento, pirólise e combustão enquanto é arrastada pelo fluxo gasoso. O char resultante é coletado em um filtro de bronze sintetizado (FCA 75), localizado na zona de pós-combustão e o gás da combustão é recolhido em um cilindro amostrador, que fica sob vácuo durante o ensaio. Tão logo atinja o filtro, 0 char é inertizado com um fluxo de $\mathrm{N}_{2}$ para evitar reações de pós-combustão. No cilindro amostrador a coleta dos gases é feita por bags para posterior análise em cromatógrafo gasoso. A atmosfera dos ensaios pode ser variada, sendo possível utilizar oxigênio, ar ou nitrogênio.

\subsection{Automação do ensaio de combustão do simulador}

A operação do simulador de PCl é executada via Controlador Lógico Programável (CLP) utilizando o supervisório Elipse E3. O sistema foi implementado pela empresa JMD Automação Industrial, com sede em Porto Alegre-RS. 


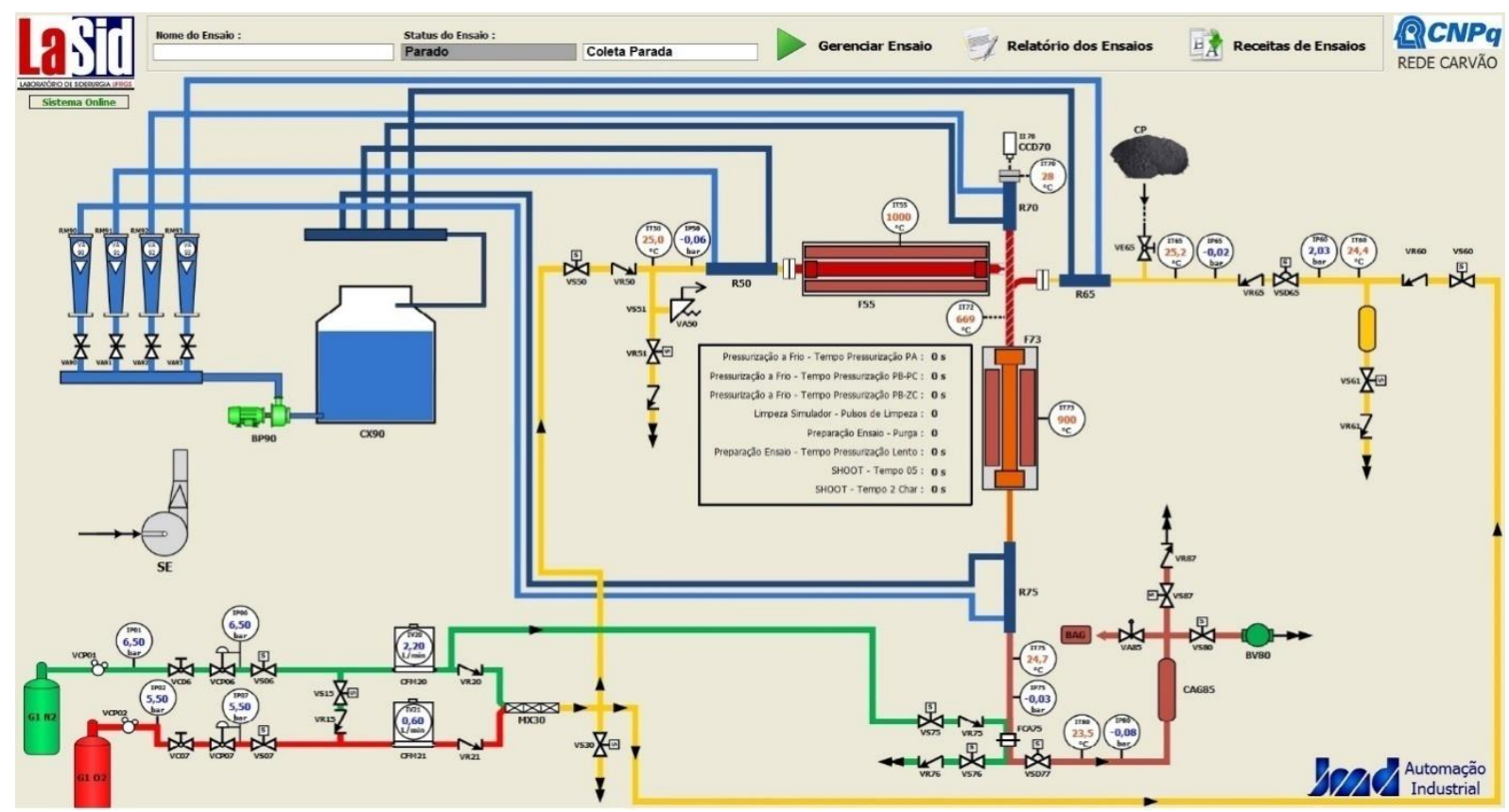

Figura 2 - imagem do supervisório desenvolvido para operação do equipamento

O simulador possui aquisição rápida e lenta de dados. A aquisição rápida é dedicada à coleta dos dados mais relevantes à análise das combustões, visto que o ensaio se dá em ms. A aquisição rápida contempla os sensores IP60, IP65, IP75 e IT72. A aquisição lenta compreende a coleta de parâmetros de pressão e temperatura em outros pontos, os quais são adquiridos na faixa de $s$.

Antes de cada ensaio é executada uma limpeza do simulador, que consiste em ensaios sem amostra para arrastar carvão e char residuais.

A automação do ensaio de combustão é realizada da seguinte forma:

- purga e vácuo: pressuriza-se o simulador com $\mathrm{O}_{2}$ até uma pressão préestabelecida com o acionamento da válvula VS07 e do CFM21. Com isso, enche-se de $\mathrm{O}_{2}$ todas as zonas do simulador, zona de baixa pressão (abertura da VS50), de alta pressão (abertura da VS60 e da VS65) e de pós-combustão (abertura da VS77). Após a pressurização, todas as válvulas são fechadas, o gás é liberado (abertura da VS65 e da VS87) e após o todo sistema é evacuado. O número de ciclos de purga e vácuo é definido pelo operador. Ao final desta etapa o supervisório solicitará a injeção da amostra. A injeção do combustível sólido (CP - carvão pulverizado) é feita através da válvula VE65, sendo esta operação realizada manualmente. O operador deve confirmar a injeção para habilitar a próxima etapa.

- pressurização da zona de alta pressão: a zona de alta pressão é pressurizada com $\mathrm{O}_{2}$ (abertura das válvulas VS07 e VS60) até ser atingida a pressão definida para o ensaio, medida pelo IP60.

- pressurização da zona de baixa pressão: a pressurização desta zona (abertura de VS07, CFM21 e VS50) ocorre lentamente, em tempo superior a $100 \mathrm{~s}$, conforme definido pelo operador, para evitar turbulências na amostra, que encontra-se depositada no tubo horizontal da região de injeção, e permitir que o gás, na região de pré-aquecimento, alcance temperartura adequada para a devida ignição da amostra de combustível a ser injetada. Esta etapa termina quando atinge-se a pressão programada (detectada pelo sensor IP65).

- repressurização da zona de alta pressão: repressuriza-se a zona de alta pressão (abertura das válvulas VS07 e VS60) para compensar possíveis vazamentos durante o tempo de pressurização da região principal do simulador medida pelo IP60. 
- pulso (one shoot): inicia a aquisição lenta de dados, que ocorre a cada 1 s. O gás pré-aquecido passa através do simulador (abertura da válvula VS76) e inicia a aquisição rápida de dados, que são coletados a cada $1 \mathrm{~ms}$. O gás entra pela zona de alta pressão (abertura da válvula VS65) durante o tempo estabelecido. A circulação de gás pré-aquecido encerra (VS76 fecha) e começa a entrar gás no cilindro amostrador (abertura da VS77) pelo intervalo de tempo estipulado. A válvula de pulso (VS65) é então fechada e a aquisição rápida de dados encerra. A seguir tem-se o fim da coleta de gás no cilindro amostrador (fechamento da VS77) e da aquisição lenta de dados.

- inertização do char: um fluxo de $\mathrm{N}_{2}$ (abertura de VS06, VS75 e VS76) inertiza o char depositado no filtro de bronze sinterizado. Ao término desta etapa o supervisório solicita a amostragem do gás de combustão, a qual é feita em bags, conforme mencionado anteriormente;

- amostragem de gás de combustão e coleta do char: o operador coleta o gás e confirma no supervisório a ação. É solicitada então a troca do filtro de bronze sinterizado, o operador remove então o filtro contendo o char e o troca por um filtro de limpeza. Esta ação também deve ser confirmada no supervisório.

O simulador possui uma câmera de alta velocidade (1200 quadros/segundo), que pode ser posicionada no topo do equipamento, possibilitando a filmagem do ensaio. Termopares de alta velocidade também podem ser colocados por essa janela para avaliar a evolução das reações.

\section{RESULTADOS E DISCUSSÃO}

Como há a possibilidade da aquisição dos parâmetros termodinâmicos do simulador ( $T$ e p), também é possível fazer modificações nos tempos determinados em cada ensaio, o que ocasionará modificação no tempo de residência da amostra na zona de combustão. Fazendo o uso desta ferramenta podem-se avaliar reações de pirólise, gaseificação e combustão. Os gráficos obtidos na aquisição de dados são utilizados para definir o início e término da reação, bem como pressão máxima de combustão e taxas de aquecimento e de pressão.

Devido ao tempo de residência do carvão ser muito pequeno, o simulador tenta ao máximo representar as condições reais de um AF neste ensaio, então na aquisição de dados o tempo é apresentado em ms.

É gerado um gráfico da variação de tempo com a temperatura e com a pressão. Os indicadores que são mostrados no gráfico são os IP60 (zona de alta pressão), IP65 (zona de baixa pressão), IP75 (zona de baixa pressão) e IT72 (ponto de encontro do carvão com o gás quente).

$\mathrm{Na}$ Figura 3 tem-se um gráfico da aquisição de dados de um ensaio em vazio, ou seja, sem amostra, visando ilustrar os parâmetros que podem ser avaliados. Nos primeiros instantes do ensaio ocorre a pressurização das zonas de alta pressão (PA) e de baixa pressão (PB) e quando estas chegam aos valores definidos ocorre 0 pulso do CP (shoot). Ao final do tiro tem-se a equalização entre as pressões. As pressões medidas pelo IP65 e IP75 são semelhantes, pois as duas pertencem à zona de baixa pressão. 


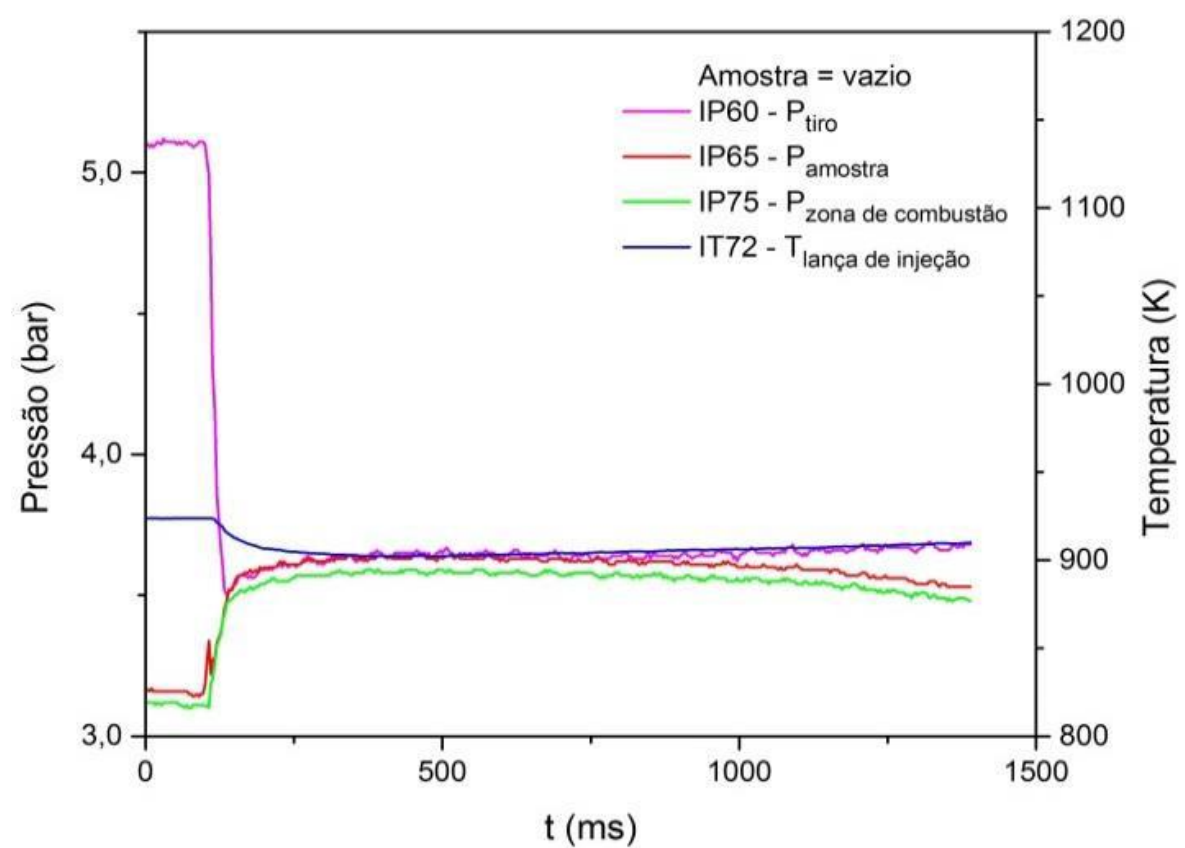

Figura 3 - Gráfico de aquisição de dados de um ensaio em vazio

Já o indicador de temperatura (IT72), posicionado no encontro do gás-sólido, mostra uma variação quando ocorre esse encontro, pois a amostra vem de uma região com gás frio. Esta troca de calor acarreta a diferença de temperatura observada no gráfico.

Quando ocorre o ensaio propriamente dito, com a presença de $\mathrm{CP}$, o gráfico será do padrão mostrado na Figura 4. A intensidade dos picos de temperatura e pressão varia de acordo com o tipo de combustível injetado. Conforme é variada a matéria carbonosa ou adicionado combustível de natureza diferente, têm-se modificações das características físicas e químicas, que resultarão em modificações nos parâmetros termodinâmicos durante o ensaio.

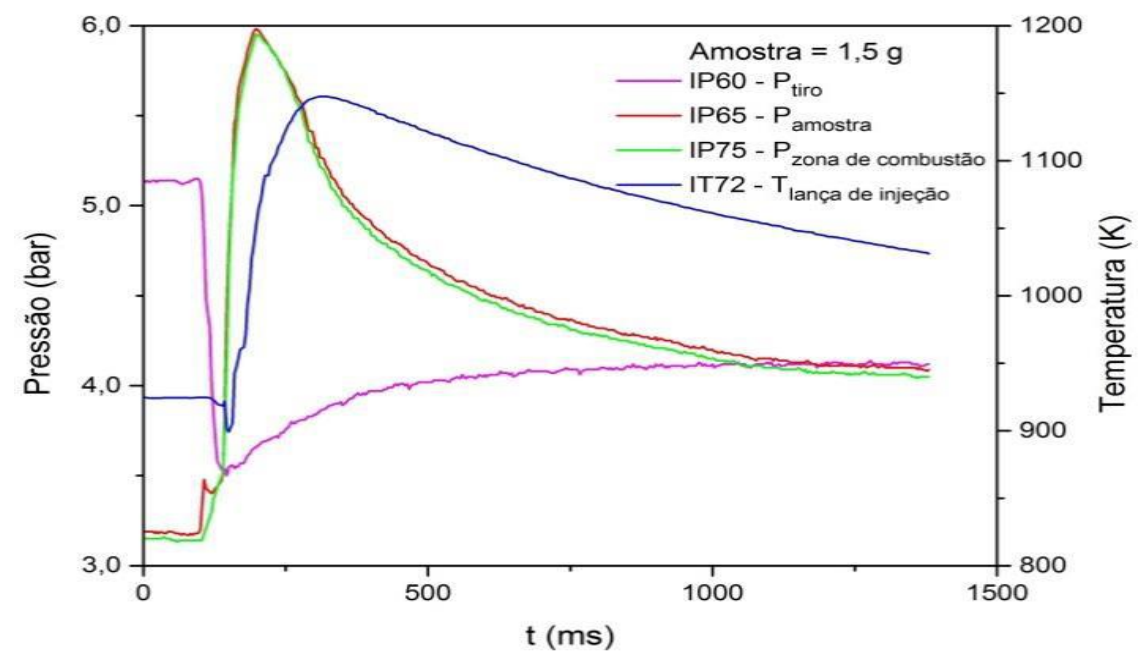

Figura 4 - Gráfico de aquisição de dados de um ensaio com 1,5g de CP

A Figura 4 apresenta o gráfico obtido pela aquisição de dados de um ensaio com 1,5 $\mathrm{g}$ de CP com granulometria entre $36-75 \mu \mathrm{m}$. Como observamos, o início do ensaio é similar ao ensaio em vazio, quando a amostra é lançada na zona de combustão. Porém neste caso verificam-se picos de pressão (IP60 e IP65) e de temperatura 
(IT72) ocasionados pela reação de combustão ultrarrápida da matéria volátil do carvão. Quanto maior a massa de amostra injetada, maior a pressão máxima de combustão atingida, pois é maior a massa de voláteis queimados. No caso de reação de combustão, a queda de temperatura inicial, além da entrada do gás frio, representa a secagem e aquecimento do carvão até o início da pirólise [3].

$\mathrm{Na}$ Figura 5 a tem-se uma imagem do reator antes de um ensaio e nas Figuras $5 \mathrm{~b}$ e 5 c são apresentadas visualizações da reação de ignição e combustão, respectivamente, obtidas durante a filmagem do ensaio.

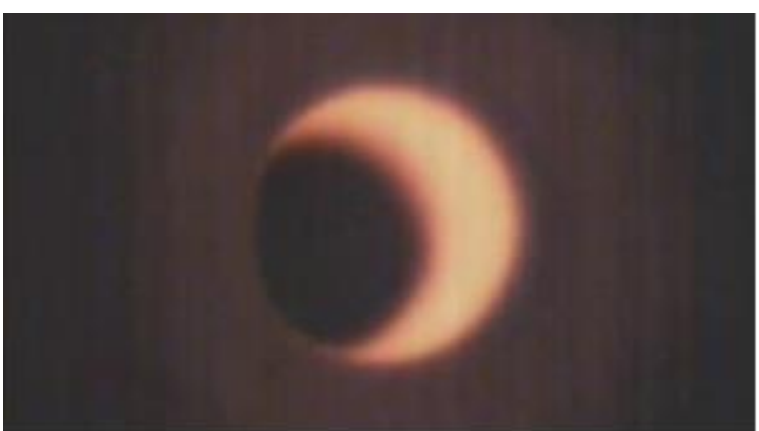

a - reator antes da injeção do CP

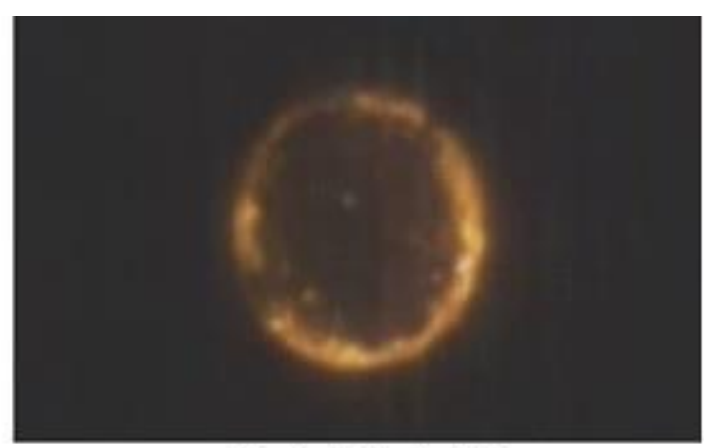

b - ignição do $\mathrm{CP}$

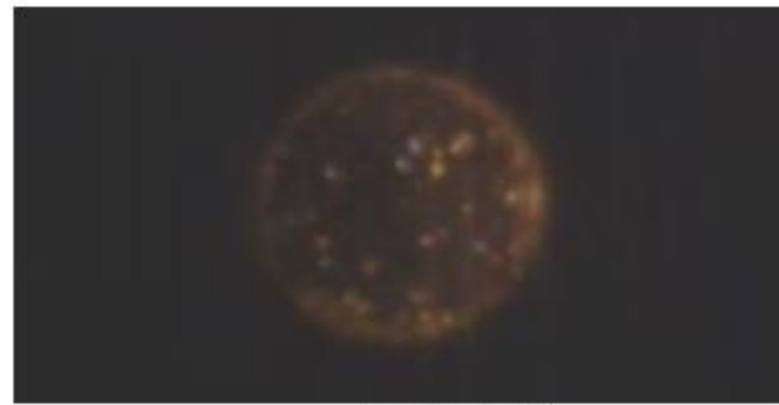

c - combustão do $\mathrm{CP}$

Figura 5 - Imagens do reator antes e durante o ensaio

Para a avaliação da eficiência de combustão é feita análise de gases e do resíduo (char). Para avaliação dos gases são realizados ensaios de cromatografia gasosa para determinar a relação $\mathrm{CO} / \mathrm{CO}_{2}$. O char é avaliado por Análise Termogravimétrica (TGA) quanto à conversão de carbono (burnout). As comparações apresentadas na Tabela 2 foram feitas com base na variação da massa do CP (carvão subbetuminoso Leão II de origem brasileira). Uma menor relação $\mathrm{CO} / \mathrm{CO}_{2}$ indica uma maior eficiência de combustão, pois quanto mais completa a combustão maior o teor de $\mathrm{CO}_{2}$ gerado. No caso das amostras avaliadas a relação $\mathrm{CO} / \mathrm{CO}_{2}$ seguiu 0 comportamento esperado: a menor relação foi observada para a menor massa $(1,0$ $\mathrm{g})$, mais fácil de queimar, e a maior relação para a maior massa $(2,0 \mathrm{~g})$, de queima mais difícil [3].

Tabela 2 - Relação $\mathrm{CO} / \mathrm{CO}_{2}$

\begin{tabular}{c|c|c|c}
\hline Massa de amostra (g) & 1,0 & 1,5 & 2,0 \\
\hline $\mathrm{CO} / \mathrm{CO}_{2}$ & 0,1 & 0,4 & 0,5 \\
\hline
\end{tabular}




\section{CONCLUSÃo}

O simulador de $\mathrm{PCl}$ projetado e desenvolvido pelo Laboratório de Siderurgia da Universidade Federal do Rio Grande do Sul (UFRGS) foi desenvolvido com o ideal de comparar e avaliar combustíveis para o AF. Apesar de o equipamento ainda estar em fase de testes pré-operacionais apresenta resultados coerentes, como por exemplo, aumento de pressão com o aumento da massa de amostra injetada. Etapas futuras incluem análise de misturas de carvões e biomassa e implantação de modo contínuo.

\section{Agradecimentos}

Agradecimentos à CNPq/Rede Carvão (processo 800265/2016-5) pelo financiamento do projeto.

\section{REFERÊNCIAS}

1 Carpenter, A, M. Use of PCl in blast furnace. London: IEA Clean Cool Centre; 2006.

2 Bennet $\mathrm{P}$, Fukushima $\mathrm{T}$. Impact of $\mathrm{PCl}$ coal quality on blast furnace operations. In: $12^{\text {th }}$ international conferece on coal science. Cairns, Qld, Australia, 2-6 Novembro 2003. Tourkley, NSW, Australia, AustralianInstituteof Energy. [acesso em 21 de maio. 2017]. Disponível em: http://www.coaltech.com.au/LinkedDocuments/Bennett_Fukushima.pdf.

3 Barbieri, C. C. T.; Machado, A. S.; Rech, R.L.; Pohlmann, Juliana Gonçalves; Osório, E.; Vilela, A. C.F. O simulador da zona de combustão do alto-forno do Laboratório de Siderurgia da UFRGS - Uma nova ferramenta na seleção de carvões para injeção. V Congresso Brasileiro de Carvão Mineral; 2017.

4 Machado, A.S.; Rech, R.L; Machado, J.G.M.S.; Vilela, A.C.F.; Osório, E. Construção de um simulador da zona de combustão do alto-forno par a avaliação de combustíveis sólidos e gasosos. Anais do IV Congresso Brasileiro de Carvão Mineral; 2013; Gramado, Brazil. p. 304-318.

5 Korthas, B. Untersuchung der verbrennungsvorgängehoherkohlenstaubmengenunterhochofennahenbedingungen und ihreauswirkungen auf die hochofenströmung. [tese de doutorado]. Aachen: Aachen Univeristy, 1987. 